\title{
Tofacitinib Versus Non-Tumor Necrosis Factor Biologics for Patients With Active Rheumatoid Arthritis
}

\author{
Yukio NAKAMURA, ${ }^{1,2}$ Takako SUZUKI, ${ }^{1}$ Hideshi YAMAZAKI, ${ }^{3}$ Hiroyuki KATO ${ }^{1}$ \\ ${ }^{1}$ Department of Orthopaedic Surgery, Shinshu University, Matsumoto, Japan \\ ${ }^{2}$ Department of Orthopaedic Surgery, Showa Inan General Hospital, Komagane, Japan \\ ${ }^{3}$ Department of Orthopaedic Surgery, Marunouchi Hospital, Matsumoto, Japan
}

\begin{abstract}
Objectives: This study aims to compare the disease status of patients with active rheumatoid arthritis (RA) after treatment with tofacitinib or nontumor necrosis factor (TNF) biologics.

Patients and methods: The study included a total of 50 RA patients (18 males, 32 females; mean age $68.3 \pm 1.3$ years; range 42 to 92 years). We prospectively and randomly enrolled 25 patients for treatment with tofacitinib (Tofa group: 10 males, 15 females; mean age $68.3 \pm 2.0$ years; range, 42 to 92 years) and 25 for treatment with non-TNF biologics (non-TNF group: 8 males, 17 females; mean age $68.3 \pm 1.7$ years; range 51 to 92 years). Mean disease activity score 28 (DAS28), C-reactive protein (CRP), clinical disease activity index (CDAI), health assessment questionnaire (HAQ)-disability index (DI), and matrix metalloproteinase-3 values were recorded at baseline and at 4, 8, and 12 months.

Results: There was a significant difference in the percent changes of DAS28, CRP and CDAl at every time point versus baseline in both treatment groups. HAQ-DI was also significantly different at every time point in both groups except for at four months in the non-TNF group.

Conclusion: Tofacitinib was well tolerated in active RA patients and exerted effects comparable to those of non-TNF biologics.

Keywords: Non-tumor necrosis factor biologics; rheumatoid arthritis; tofacitinib.
\end{abstract}

The short-term efficacy of tofacitinib (Tofa) was reported to be as strong as that of the tumor necrosis factor (TNF) inhibitor adalimumab in the ORAL standard trial. ${ }^{1}$ Tofa is frequently used in Japan for rheumatoid arthritis (RA) patients who are inadequately responsive to methotrexate (MTX) of $>8 \mathrm{mg} /$ week or who are unresponsive to biologics. However, there exist few clinical studies on Tofa with respect to its effectiveness and safety in patients with active RA despite its recognition as a treatment option, ${ }^{2}$ nor are there any reports comparing Tofa and non-TNF agents such as tocilizumab and abatacept in active RA patients to date. We routinely prescribe biologics or Tofa at our institutions for MTX-unresponsive patients and switch the drug if it is ineffective. In our study, oral Tofa $10 \mathrm{mg} /$ day was orally administered to biologic- or MTX-unresponsive patients. Therefore, in this study, we aimed to compare the disease status of patients with active RA after treatment with Tofa or non-TNF biologics.

\section{PATIENTS AND METHODS}

This prospective randomized study was conducted at Shinshu University School of Medicine and Showa Inan General Hospital between June 2014 and June 2016 and included a total of 50 RA patients (18 males, 32 females; mean age $68.3 \pm 1.3$ years; range 42 to 92 years). We enrolled the patients into two groups of 25 with active RA for either Tofa treatment (Tofa group) (10 males,

Received: January 23, 2017 Accepted: August 11, 2017 Published online: October 13, 2017

Correspondence: Yukio Nakamura, MD. Department of Orthopaedic Surgery, Shinshu University, 3908621 Matsumoto, Japan.

Tel: 263372659 e-mail:yxn14@aol.jp

(02018 Turkish League Against Rheumatism. All rights reserved. 
15 females; mean age $68.3 \pm 2.0$ years; range 42 to 92 years) or non-TNF biologics treatment (non-TNF group) (8 males, 17 females; mean age $68.3 \pm 1.7$ years; range 51 to 92 years). Three patients dropped out of the Tofa group because of serious infections and five were lost in the nonTNF group due to insufficient data. Consequently, 22 patients in the Tofa group and 20 in the nonTNF group (tocilizumab: 8, abatacept: 12) were further analyzed for remission rate. The study protocol was approved by the Shinshu University School of Medicine and Showa Inan General Hospital Ethics Committee. A written informed consent was obtained from each patient. The study was conducted in accordance with the principles of the Declaration of Helsinki. This study was registered in the Clinical Trials database (IRB approval number: NCT02157012) in May 2014.

Overall, mean age was $67.2 \pm 1.7$ years and $68.3 \pm 1.2$ years in the Tofa and non-TNF groups, respectively. Mean disease duration was $3.4 \pm 0.7$ years and $3.5 \pm 1.0$ years in the Tofa and non-TNF groups, respectively. All patients had prolonged moderate disease activity score 28-C-reactive protein (DAS28-CRP) scores, indicating that the prior treatment had been ineffective (Table 1). The subsequently analyzed MTX-unresponsive or biologics-naïve patients were summarized in Table 2. Mean age of these patients was $67.8 \pm 1.6$ years and $67.8 \pm 3.3$ years in the Tofa and nonTNF groups, respectively. Mean disease duration was $3.4 \pm 0.1$ years and $3.3 \pm 0.3$ years in the Tofa and non-TNF groups, respectively.

In the Tofa group, 6 of the 22 patients were changed to Tofa because of secondary failure of previous biologics. Six patients had received pretreatment biologics (infliximab: 1, adalimumab: 2, etanercept: 2 , certolizumab pegol: 1). Tofa was added for the remaining 16 biologics-naïve patients after treatment with MTX $>8 \mathrm{mg}$ for more than 3 months had proved ineffective. In the non-TNF group, biologics had been changed from other biologics in 5 patients exhibiting primary or secondary failure. Five patients had received pretreatment biologics (infliximab: 2, adalimumab: 1 , etanercept: 2), while the remaining 15 patients were biologics-naïve after treatment with MTX $>8 \mathrm{mg}$ for more than 3 months was judged ineffective.

Oral Tofa was administered $5 \mathrm{mg}$ twice daily during the observational period. Remission was evaluated based on DAS28-CRP scores before and at 12 months of therapy. The diagnosis and treatment of RA in this study were conducted in accordance with the 2010 American College of Rheumatology (ACR)/European League Against Rheumatism (EULAR) classification system. ${ }^{3}$

Laboratory matrix metalloproteinase-3 (MMP-3) (Sekisui Medical Co., Ltd., Tokyo, Japan) was assessed before and at 4, 8, and 12 months after starting treatment. We also recorded the percent changes in DAS28-CRP,

Table 1. Characteristics of overall patients in tofacitinib and non-tumor necrosis factor biologics groups

\begin{tabular}{|c|c|c|c|c|c|c|c|}
\hline \multirow[t]{2}{*}{ Characteristic } & \multicolumn{3}{|c|}{ Tofa group $(n=22)$} & \multicolumn{3}{|c|}{ Non-TNF group $(n=20)$} & \multirow[b]{2}{*}{$p$} \\
\hline & $\mathrm{n}$ & $\%$ & Mean \pm SE & $\mathrm{n}$ & $\%$ & Mean \pm SE & \\
\hline Age (year) & & & $67.2 \pm 1.7$ & & & $68.3 \pm 1.2$ & 0.5978 \\
\hline \multicolumn{8}{|l|}{ Sex } \\
\hline Female & 13 & & & 17 & & & \\
\hline Male & 9 & & & 3 & & & \\
\hline Body mass index $\left(\mathrm{kg} / \mathrm{m}^{2}\right)$ & & & $21.8 \pm 0.8$ & & & $20.6 \pm 1.1$ & 0.3696 \\
\hline Disease duration (year) & & & $3.4 \pm 0.7$ & & & $3.5 \pm 1.0$ & 0.9556 \\
\hline Bio-naïve & 16 & 72.7 & & 15 & 75.0 & & \\
\hline Methotrexate & 19 & 86.4 & & 13 & 65.0 & & \\
\hline Methotrexate, dose (mg/week) & & & $8.8 \pm 0.8$ & & & $8.8 \pm 1.1$ & 0.9798 \\
\hline Methotrexate, period (months) & & & $13.2 \pm 0.7$ & & & $15.9 \pm 1.5$ & 0.1491 \\
\hline Glucocorticoid & 8 & 36.4 & & 9 & 45.0 & & \\
\hline Glucocorticoid, dose (mg/day) & & & $5.2 \pm 0.5$ & & & $5.5 \pm 0.7$ & 0.7339 \\
\hline Disease activity score 28 -C-reactive protein & & & $4.2 \pm 0.1$ & & & $4.2 \pm 0.2$ & 0.7895 \\
\hline Clinical disease activity index & & & $19.7 \pm 2.2$ & & & $18.7 \pm 2.2$ & 0.7448 \\
\hline Health assessment questionnaire-disability index & & & $0.7 \pm 0.2$ & & & $0.7 \pm 0.2$ & 0.9070 \\
\hline Metalloproteinase-3 (IU/mL) & & & $154.0 \pm 48.0$ & & & $150.4 \pm 28.6$ & 0.9498 \\
\hline
\end{tabular}




\begin{tabular}{|c|c|c|c|c|c|c|c|}
\hline \multirow[t]{2}{*}{ Characteristic } & \multicolumn{3}{|c|}{ Tofa group $(n=16)$} & \multicolumn{3}{|c|}{ Non-TNF group $(n=15)$} & \multirow[b]{2}{*}{$p$} \\
\hline & $\mathrm{n}$ & $\%$ & Mean \pm SE & $\mathrm{n}$ & $\%$ & Mean \pm SE & \\
\hline Age (year) & & & $67.8 \pm 1.6$ & & & $67.8 \pm 3.3$ & 0.9944 \\
\hline \multicolumn{8}{|l|}{ Sex } \\
\hline Female & 13 & & & 12 & & & \\
\hline Male & 3 & & & 3 & & & \\
\hline Body mass index $\left(\mathrm{kg} / \mathrm{m}^{2}\right)$ & & & $21.9 \pm 1.1$ & & & $20.2 \pm 1.6$ & 0.4061 \\
\hline Disease duration (year) & & & $3.4 \pm 0.1$ & & & $3.3 \pm 0.3$ & 0.8323 \\
\hline Bio-naïve & 16 & 100 & & 15 & 100 & & \\
\hline Methotrexate & 16 & 100 & & 15 & 100 & & \\
\hline Methotrexate, dose (mg/week) & & & $9.8 \pm 0.7$ & & & $10.0 \pm 1.0$ & 0.8760 \\
\hline Methotrexate, period (months) & & & $14.0 \pm 1.2$ & & & $14.4 \pm 0.6$ & 0.7687 \\
\hline Glucocorticoid & 3 & 18.8 & & 2 & 13.3 & & \\
\hline Glucocorticoid, dose (mg/day) & & & $4.2 \pm 0.8$ & & & $4.3 \pm 0.3$ & 0.8645 \\
\hline Disease activity score 28-C-reactive protein & & & $4.0 \pm 0.1$ & & & $3.9 \pm 0.4$ & 0.7098 \\
\hline Clinical disease activity index & & & $16.3 \pm 1.9$ & & & $15.1 \pm 1.6$ & 0.6619 \\
\hline Health assessment questionnaire-disability index & & & $0.7 \pm 0.2$ & & & $0.6 \pm 0.3$ & 0.7511 \\
\hline Metalloproteinase-3 (IU/mL) & & & $161.1 \pm 20.4$ & & & $169.0 \pm 29.5$ & 0.8406 \\
\hline
\end{tabular}

clinical disease activity index (CDAI) instead of simplified disease activity index due to the use of tocilizumab, and health assessment questionnaire-disability index (HAQ-DI) for all patients at set time points.

We ultimately compared the efficiency of Tofa $(n=16)$ with that of non-TNF $(n=15)$ in biologicsnaive subjects who were non-responsive to more than 3 months of MTX $>8 \mathrm{mg}$ treatment (Table 2). We analyzed if the treatments were effective and whether there existed differences between Tofa and non-TNF groups.

\section{Statistical analysis}

Comparisons between the DAS28-CRP, CDAI, HAQ-DI, and MMP-3 scores of both groups versus pre-treatment at each measuring point were assessed with the corresponding Bonferroni multiple comparisons procedure. Comparisons of DAS28-CRP, CDAI, HAQ-DI, and MMP-3 between the groups at each time point were performed by Welch's t-test. A value of $p<0.05$ indicated a statistically significant difference. Data were expressed as the mean value \pm standard error.

\section{RESULTS}

There were no significant differences in age or mean affected period between the groups.
Thirteen of 22 patients (59.1\%) in the Tofa group and 11 of 20 patients (55.0\%) in the non-TNF group had entered remission at 12 months based on DAS28-CRP data.

Comparisons of the MTX-unresponsive, biologics-naïve Tofa and non-TNF groups (Figure 1, Table 2) revealed that the percent changes of DAS28-CRP and CDAI displayed significant differences at every time point in both groups compared with pre-treatment values (Figure 1a, b). The percent change of HAQ-DI showed significant differences at every time point in both groups compared with baseline values except at 4 months in the non-TNF group (Figure 1c). The percent change of DAS28-CRP compared with baseline exhibited significant decreases at 12 months of $-46.5 \pm 7.0 \%$ in the Tofa group and $-44.7 \pm 9.4 \%$ in the non-TNF group. These percent changes were $-61.6 \pm 5.5 \%$ and $-60.0 \pm 9.0 \%$, respectively, for CDAI and $-60.0 \pm 12.4 \%$ and $-57.5 \pm 15.8 \%$, respectively, for HAQ-DI. The inflammatory disease index MMP-3 decreased remarkably and comparatively by $24.7 \%$ in the Tofa group and $24.9 \%$ in the non-TNF group at 12 months. There were no significant differences in the percent change of MMP-3 compared with pre-treatment values for either group, although levels tended to decrease (Figure 1d). 
(a)

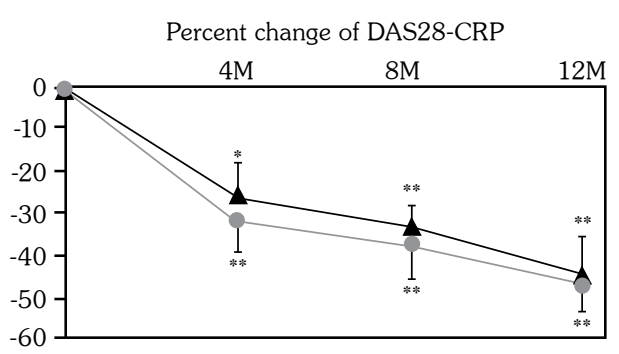

(b)

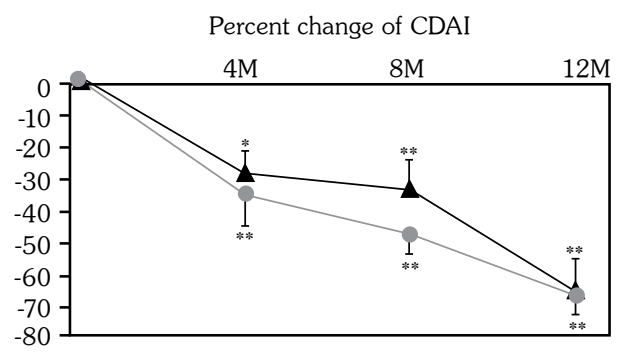

(c)

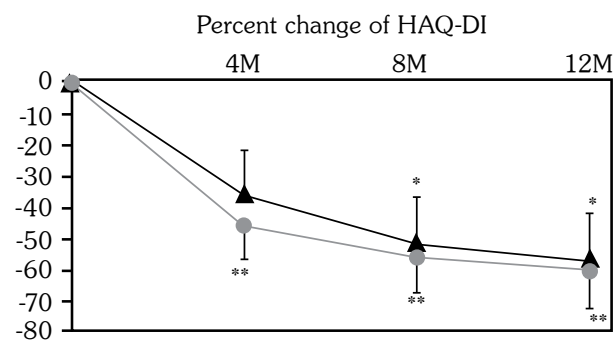

(d)

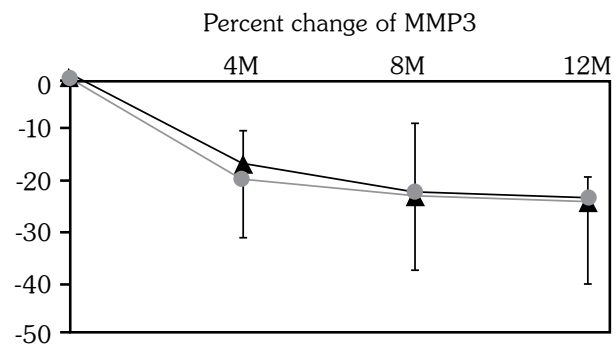

Figure 1. Percent changes in average disease activity score-28 C-reactive protein (DAS28-CRP) (a), clinical disease activity index (CDAI) (b), health assessment questionnairedisability index (HAQ-DI) (c), and matrix metalloproteinase-3 (MMP-3) (d) before and at 4, 8, and 12 months after starting tofacitinib (Tofa group) or non-tumor necrosis factor (non-TNF) biologics (non-TNF group) treatment in Japanese patients with active rheumatoid arthritis. Circles: Tofacitinib, triangles: Non-TNF biologics. "Significant difference of $p<0.05$; ${ }^{* *}$ Significant difference of $\mathrm{p}<0.01$; Values are expressed as mean \pm standard error.

\section{DISCUSSION}

This unique comparative study on the effectiveness and clinical safety of Tofa and non-TNF biologics in Japanese patients with active RA was conducted during a course of over 1 year of treatment. Disease state and laboratory results showed marked improvements during the observational period in both groups. To our knowledge, this is the first study comparing Tofa versus non-TNF biologics in this type of patient population.

Rheumatoid arthritis is characterized by chronic synovial inflammation due to unknown causes. In clinical trials, molecular-targeted therapies produced a significant reduction in RA symptoms and provided pain relief in patients with active RA. ${ }^{4}$ Conventional synthetic diseasemodifying anti-rheumatic drugs (DMARDs), biologic DMARDs (bDMARDs), and now Tofa, a targeting synthetic DMARD, provide a wide range of treatment options for this condition. ${ }^{5}$
Cohen et al. ${ }^{6}$ analyzed the efficacy and safety of Tofa in RA patients in the United States and abroad. In their report, pooled phase III data from patients receiving 5 or $10 \mathrm{mg}$ Tofa or placebo twice daily confirmed the efficacy and safety outcomes at 3 and 12 months, respectively. Lundquist et al. ${ }^{7}$ reported that numerous phase II and III trials proved Tofa to be safe and effective as a monotherapy or in combination with MTX or other non-biologic DMARDs. Moreover, the clinical efficacy of Tofa and MTX was shown to be equivalent to that of adalimumab in patients with RA. ${ }^{4}$ Even patients who did not show clinical improvement with combination non-biologic DMARD and MTX therapy exhibited significantly inhibited structural damage. ${ }^{5}$ Taken together, these data indicate that Tofa is a good oral DMARD candidate for treating patients with active RA. In this study, 3 of 25 patients (12.0\%) discontinued Tofa because of signs of infection (e.g., cough, fever, nasal mucus discharge), which was comparable with incidences in 
previous studies. The remaining $22 \mathrm{RA}$ patients displayed no visible adverse effects.

Recently, the 2016 EULAR recommendations for the management of RA stated that Tofa could be considered a first-line molecular-targeted therapy. Moreover, the drug is orally administered, which is advantageous for drug adherence in patients who dislike subcutaneous or intravenous injection. ${ }^{8}$ Iwamoto et al. ${ }^{9}$ very recently reported that Tofa was effective even without MTX use or after switching from tocilizumab after 24 weeks of treatment. In this study, we compared the effectiveness of Tofa and non-TNF biologics in biologics-naïve groups that had been unresponsive to MTX > $8 \mathrm{mg}$ for more than 3 months. Moreover, Tanaka et al. ${ }^{2}$ evaluated Tofa versus placebo for treatment of active RA in Japanese patients with an inadequate response to DMARDs in a clinical trial and found that the drug produced dosedependent ACR 20\% improvement response criteria and reduced disease activity. In addition, the therapeutic efficacy of Tofa was observed to be as strong as that of TNF inhibitors in active RA patients. ${ }^{10}$ The percent changes of the clinical disease activity indices DAS28-CRP and CDAI were significantly improved by $-46.5 \%$ and $-61.6 \%$, respectively, in the Tofa group and $-44.7 \%$ and $-60.0 \%$, respectively, in the nonTNF group at 12 months of therapy, with no significant differences in improvements from pre-treatment between the groups at any time point. Concerning the functional disability index HAQ-DI, percent change was significantly decreased by $60.0 \%$ in the Tofa group and $57.5 \%$ in the non-TNF group at 12 months, again with no significant difference in improvements from baseline between the groups. The inflammatory disease index MMP-3 decreased remarkably and comparatively by $24.7 \%$ in the Tofa group and $24.9 \%$ in the non-TNF group at 12 months. Based on previous reports ${ }^{10}$ and our findings, Tofa is a clinically and functionally effective drug that is not only similar to non-TNF agents, but also to overall biologics in active RA patients in Japan.

To our knowledge, the present study on the effectiveness and adverse effects associated with Tofa versus non-TNF biologics was the first of its kind in Japan. It showed that: (i) in 16 biologicsnaïve patients who received Tofa therapy, RA manifestations were greatly alleviated; (ii) among
25 patients taking Tofa, there were 3 dropout patients due to infection-like symptoms, which promptly disappeared after Tofa cessation; and (iii) RA-associated conditions in all 6 of the patients who switched from biologics to Tofa were markedly ameliorated as well.

The limitations of this study were its small sample size, short follow-up period, absence of controls, and relatively short RA disease duration.

In conclusion, Tofa was well tolerated in Japanese patients with active RA and produced remarkable improvements in laboratory RA indicators that were comparable to those imparted by non-TNF biologics. Future studies with larger sample size and longer observational period are warranted.

\section{Declaration of conflicting interests}

The authors declared no conflicts of interest with respect to the authorship and/or publication of this article.

\section{Funding}

The authors received no financial support for the research and/or authorship of this article.

\section{REFERENCES}

1. van Vollenhoven RF, Fleischmann R, Cohen S, Lee EB, García Meijide JA, Wagner S, et al. Tofacitinib or adalimumab versus placebo in rheumatoid arthritis. N Engl J Med 2012;367:508-19.

2. Tanaka Y, Takeuchi T, Yamanaka H, Nakamura $\mathrm{H}$, Toyoizumi S, Zwillich S. Efficacy and safety of tofacitinib as monotherapy in Japanese patients with active rheumatoid arthritis: a 12-week, randomized, phase 2 study. Mod Rheumatol 2015;25:514-21.

3. van der Linden MP, Knevel R, Huizinga TW, van der Helm-van Mil AH. Classification of rheumatoid arthritis: comparison of the 1987 American College of Rheumatology criteria and the 2010 American College of Rheumatology/European League Against Rheumatism criteria. Arthritis Rheum 2011;63:37-42.

4. Koike T. Treatment of rheumatoid arthritis by molecular-targeted agents: efficacy and limitations. J Orthop Sci 2015;20:951-7.

5. Takeuchi T, Tanaka Y, Yamanaka H, Amano K, Nagamine R, Park W, et al. Efficacy and safety of olokizumab in Asian patients with moderate-to-severe rheumatoid arthritis, previously exposed to anti-TNF therapy: Results from a randomized phase II trial. Mod Rheumatol 2016;26:15-23. 
6. Cohen SB, Koenig A, Wang L, Kwok K, Mebus CA, Riese R, et al. Efficacy and safety of tofacitinib in US and non-US rheumatoid arthritis patients: pooled analyses of phase II and III. Clin Exp Rheumatol 2016;34:32-6.

7. Lundquist LM, Cole SW, Sikes ML. Efficacy and safety of tofacitinib for treatment of rheumatoid arthritis. World J Orthop 2014;5:504-11.

8. Smolen JS, Landewé R, Bijlsma J, Burmester G, Chatzidionysiou $\mathrm{K}$, Dougados $\mathrm{M}$, et al. EULAR recommendations for the management of rheumatoid arthritis with synthetic and biological diseasemodifying antirheumatic drugs: 2016 update. Ann Rheum Dis 2017;76:960-977.

9. Iwamoto N, Tsuji S, Takatani A, Shimizu T, Fukui S, Umeda M, et al. Efficacy and safety at 24 weeks of daily clinical use of tofacitinib in patients with rheumatoid arthritis. PLoS One 2017; 12:e0177057.

10. Tanaka Y. Recent progress and perspective in JAK inhibitors for rheumatoid arthritis: from bench to bedside. J Biochem 2015;158:173-9. 